\title{
The use of BMAD in simulating transverse and longitudinal dynamics in RHIC
}

\author{
Henry Lovelace III
}

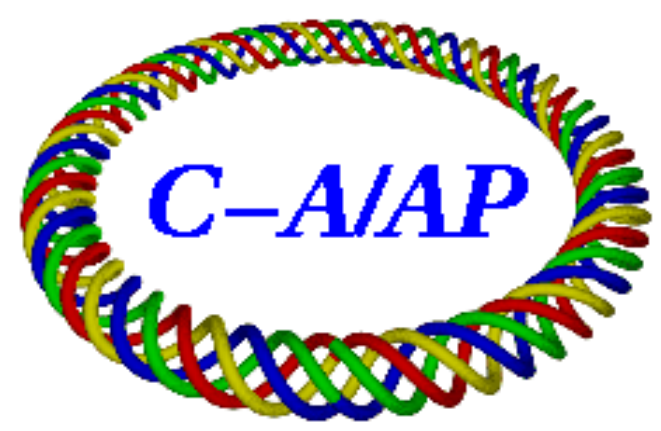

Collider-Accelerator Department Brookhaven National Laboratory Upton, N.Y. 11973

\section{U.S. Department of Energy Office of Science, Office of Nuclear Physics}

Notice: This document has been authorized by employees of Brookhaven Science Associates, LLC under Contract No. DE-SC0012704 with the U.S. Department of Energy. The United States Government retains a nonexclusive, paid-up, irrevocable, world-wide license to publish or reproduce the published form of this document, or allow others to do so, for United States Government purposes. 


\section{DISCLAIMER}

This report was prepared as an account of work sponsored by an agency of the United States Government. Neither the United States Government nor any agency thereof, nor any of their employees, nor any of their contractors, subcontractors, or their employees, makes any warranty, express or implied, or assumes any legal liability or responsibility for the accuracy, completeness, or any third party's use or the results of such use of any information, apparatus, product, or process disclosed, or represents that its use would not infringe privately owned rights. Reference herein to any specific commercial product, process, or service by trade name, trademark, manufacturer, or otherwise, does not necessarily constitute or imply its endorsement, recommendation, or favoring by the United States Government or any agency thereof or its contractors or subcontractors. The views and opinions of authors expressed herein do not necessarily state or reflect those of the United States Government or any agency thereof. 
The Use of BMAD in Simulating Transverse and Longitudinal Dynamics in RHIC

\author{
Henry Lovelace III \\ C-AD
}

Brookhaven National Laboratory

Abstract

In accelerator physics, models of a given machine are used to predict the behaviors of the beam, magnets, and radiofrequency cavities. The use of the computational model has become wide spread to ease the development period of the accelerator lattice. There are various programs that are used to create lattices and run simulations of both transverse and longitudinal beam dynamics. The programs include Methodical Accelerator Design(MAD) MAD8, MADX, Zgoubi, Polymorphic Tracking Code (PTC), and many others. In this discussion the BMAD (Baby Methodical Accelerator Design) is presented as an additional tool in creating and simulating accelerator lattices for the study of beam dynamics in the Relativistic Heavy Ion Collider (RHIC).

\title{
Introduction
}

A sequence of elements and there attributes that a particle traverses is known as a lattice. In closed geometry lattices, such as storage rings and synchrotrons, the FODO (focusing, drift, defocusing, drift) lattice system is commonly used. Variations may occur in the lattice, however, the derivation of the closed orbit and other transverse beam dynamics still hold true. The local reference orbit describes the coordinate system that is used for BMAD particle tracking.

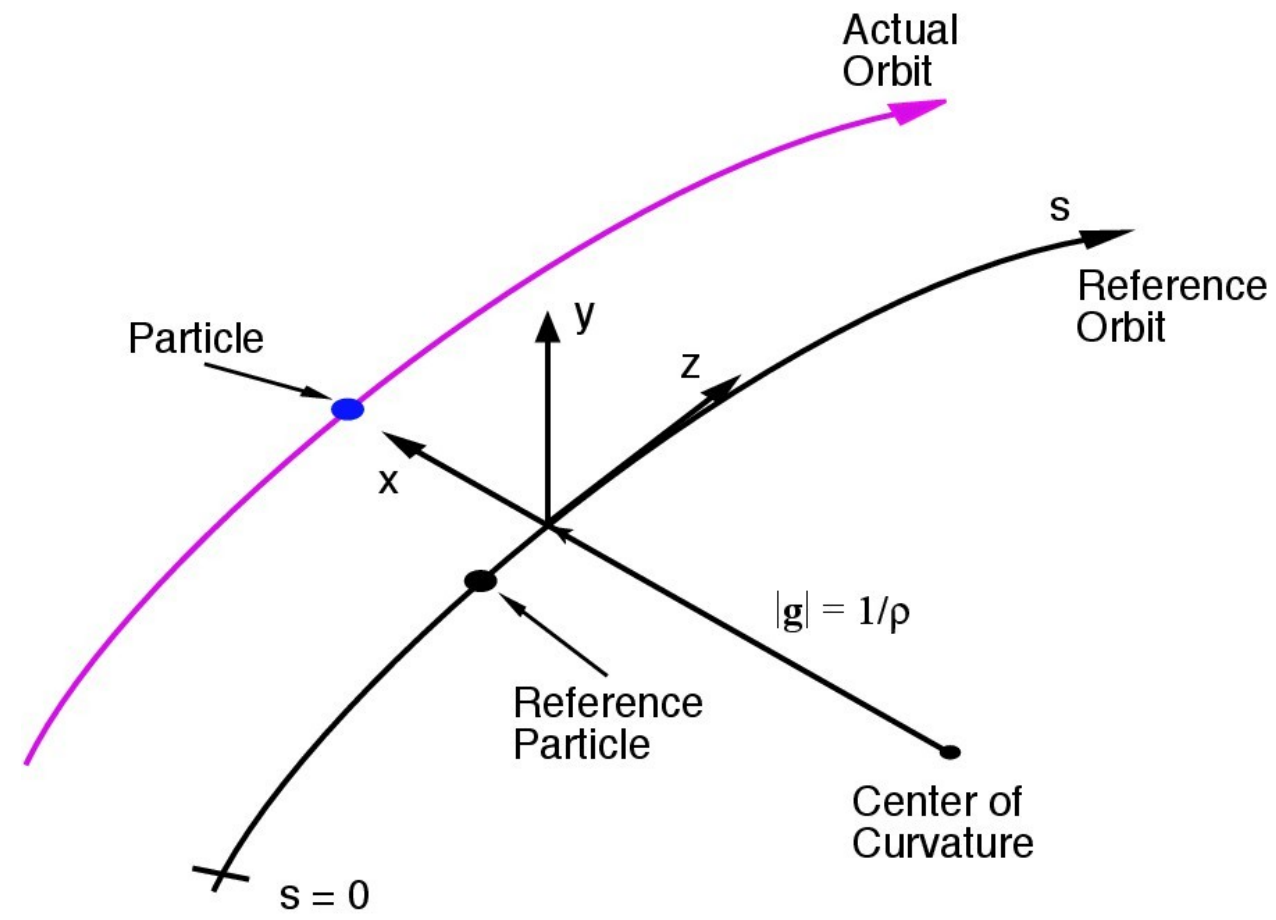

Hill's equation, which was first used to describe the three body problem of orbits of the Moon around the Earth, are now used to describe the motion of a particle in an accelerator. Hill's equation is a second order ordinary differential equation, 


$$
x^{\prime \prime}+K_{x}(s) x=0
$$

where

$$
\begin{gathered}
x^{\prime} \equiv d x / d s \\
K_{x}(s) \equiv B^{\prime} /(B \rho)+\rho^{-2} \\
B^{\prime} \equiv \partial B_{y} / \partial x
\end{gathered}
$$

where B is the magnetic field strength, and $\rho$ the radius of the circular lattice. The solution of Hill's equation in phase amplitude format is

$$
\begin{gathered}
x(s)=A \sqrt{\beta(s)} \cos (\varphi(s)+\delta) \\
x(s)=\sqrt{\varepsilon \beta(s)} \cos \left(\varphi(s)-\varphi_{0}\right)
\end{gathered}
$$

where $\varepsilon$ is the emittance, $\beta$ is the amplitude of the oscillation, $\varphi$ is the phase and $\varphi_{0}$ phase offset. Taking the derivative of the solution we have,

$$
\begin{gathered}
x^{\prime}(s)=\frac{-A}{\sqrt{\beta(s)}}[\alpha(s) \cos (\varphi(s)+\delta)+\sin (\varphi(s)+\delta)] \\
\alpha(s) \equiv-\beta^{\prime}(s) / 2
\end{gathered}
$$

Rewriting Hill's equation in terms of A we have the Courant-Snyder invariant,

$$
\varepsilon=A^{2}=\gamma(s) x^{2}+2 \alpha(s) x x^{\prime}+\beta x^{\prime 2}
$$

we have $\alpha, \beta$, and $\gamma$ which, including $\varepsilon$, are the ellipse parameters. Note that BMAD does not use Hill's equation for tracking. BMAD uses the six dimensional phase space coordinates $\left(\mathrm{x}, \mathrm{p}_{\mathrm{x}}, \mathrm{y}, \mathrm{p}_{\mathrm{y}}, \mathrm{z}, \mathrm{p}_{\mathrm{z}}\right)$ with transfer maps. The PTC /FPP engine within BMAD allows the calculation of both Taylor maps (Truncated Power Series Algebra) and Lie algebra operations. FPP is a package that is used to produce and analize the Taylor approximation of the map. BMAD has the ability to track particles using symplectic and non-symplectic maps. An example of how to calculate the Courant-Snyder invariant from the transfer map follows.

Let $M$ be a the one turn map of somewhere in the lattice.

$$
M=\left[\begin{array}{ll}
a & b \\
c & d
\end{array}\right] \quad a d-b c=1
$$

There is a symplectic matrix $S$ that satisfies this condition,

$$
M=S R S^{-1}
$$




$$
\begin{gathered}
R=\left[\begin{array}{cc}
\cos (v) & \sin (v) \\
-\sin (v) & \cos (v)
\end{array}\right] \\
S^{-1}=\left[\begin{array}{ll}
S_{22} & -S_{12} \\
S_{21} & -S_{11}
\end{array}\right]
\end{gathered}
$$

$M$ can now be written as

$$
\begin{gathered}
M=\cos (\mu) I+\sin (\mu)\left[\begin{array}{cc}
-S_{11} S_{21}-S_{12} S_{22} & S_{11}^{2}+S_{12}^{2} \\
-S_{21}^{2}-S_{22}^{2} & S_{11} S_{21}+S_{12} S_{22}
\end{array}\right] \\
M=\left[\begin{array}{cc}
\cos (\mu)+\alpha \sin (\mu) & \beta \sin (\mu) \\
-\gamma \sin (\mu) & \cos (\mu)-\alpha \sin (\mu)
\end{array}\right]
\end{gathered}
$$

This is the de Moivre formula for the one degree of freedom sympletic model. The angle of the rotation is known as the fractional tune when measured in revolutions.

$$
\operatorname{det} M=\gamma \beta-\alpha^{2}=1
$$

The Courant-Snyder invariant can now be written as

$$
\varepsilon=\left(S_{21}^{2}+S_{22}^{2}\right) x^{2}+2\left(-S_{11} S_{21}-S_{12} S_{22}\right) x p+\left(S_{11}^{2}+S_{22}^{2}\right) p^{2}
$$

The operating point of a circular accelerator is defined as

$$
v=\frac{1}{2 \pi} \oint \frac{d s}{\beta(s)}
$$

where $s$ is the longitudinal coordinate position in the beam pipe. The chromaticity of the beam is defned as

$$
\xi=\frac{\Delta v}{\Delta p / p}
$$

where $\mathrm{p}$ is the longitudinal momentum of the particle. The revolution frequency of the particle is defined as the

$$
f_{\text {rev }}=\frac{1}{T_{o}}=\frac{c \beta}{C}
$$


where $T_{0}$ is the period of one revolution, $C$ is the circumfernce of the accelerator, $c$ is the speed of light, and $\beta$ is the ratio of particle velocity and speed of light. The RF-frequency is determined by

$$
f_{r f}=h f_{r e v}
$$

where $\mathrm{h}$ is the harmonic number. From the dipole bending, dipersion occurs which causes horizontal orbit deviations. Dispersion can be defined as

$$
\Delta x=D(x, s)\left(\Delta p / p_{0}\right)
$$

where $\mathrm{x}$ is the orbit position and $\mathrm{D}$ is the dispersion function. In BMAD, the dispersion calculation is taken a step further with the use of the define dispersion equations

$$
\begin{gathered}
\eta_{x}(s)=\frac{d x}{d p_{z}} \quad \eta_{y}(s)=\frac{d y}{d p_{z}} \quad \eta_{z}(s)=\frac{d z}{d p_{z}} \\
\eta_{x}^{\prime}(s) \equiv \frac{d \eta_{x}}{d s}=\frac{d x^{\prime}}{d p_{z}} \quad \eta_{y}^{\prime}(s) \equiv \frac{d \eta_{y}}{d s}=\frac{d y^{\prime}}{d p_{z}}
\end{gathered}
$$

where $\eta$ and $\eta$ ' describe the dispersion and dispersion derivative.

The energy of the particle beam is defined by the product of the species mass in $\mathrm{GeV}$ and the Lorentz factor. The momentum of the beam can be calculated using the relation,

$$
p=\frac{E_{t o t} * \sqrt{\left(1.0-\left(m c^{2} / E_{t o t}\right)^{2}\right)}}{c}
$$

where $\mathrm{p}$ is momentum, $\mathrm{m}$ is the mass of the particle, $\mathrm{E}_{\text {tot }}$ is the total beam energy, and $\mathrm{c}$ is the speed of light. It is useful for heavy ions to describe both the energy and momentum per nucleon. In a bunch, the particles differ in momenta. The closed orbit differs due to the difference in momenta and can be expressed by a factor,

$$
\alpha_{p}=\frac{\frac{\Delta C}{C}}{\frac{\Delta p}{p_{0}}} \quad \alpha_{p}=\frac{1}{C} \int_{s}\left\langle\frac{D}{\rho}\right\rangle d s \approx \frac{\sum_{(\text {all dipole })} D \theta_{i}}{C}
$$

where the $\mathrm{D}$ is the dipersion, $\rho$ is the bending radius, and $\theta_{\mathrm{i}}$ is the bending angle. Both the bending radius and angle are attributes of the dipole. Transition gamma can then be defined as,

$$
\gamma_{t} \equiv \frac{1}{\sqrt{\alpha_{p}}}
$$


and the amount of slippage is,

$$
\eta=\frac{1}{\gamma_{t}^{2}}-\frac{1}{\gamma^{2}}
$$

A subroutine has been written to calculate this momentum compaction in a closed geometry lattice.

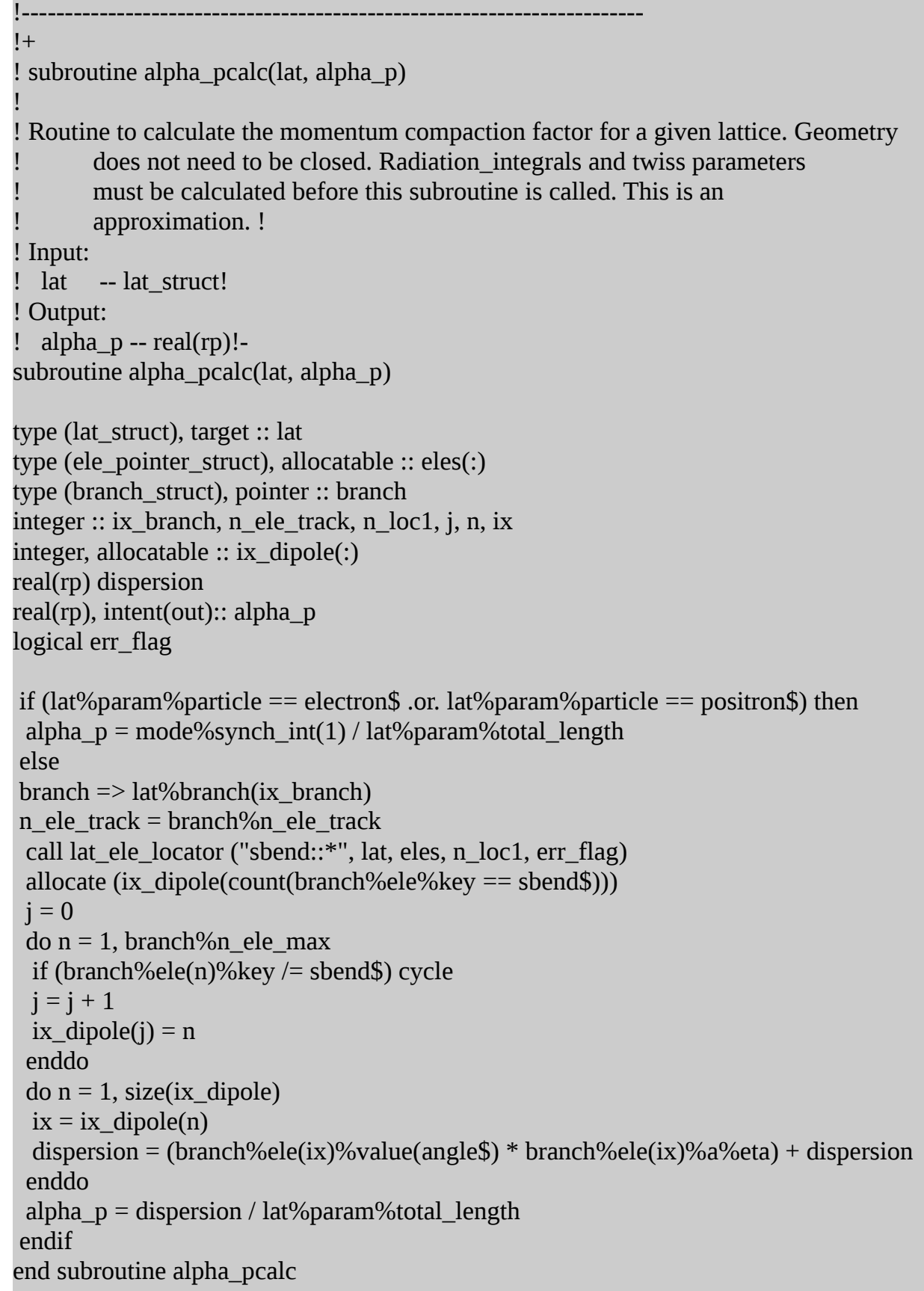

Closed orbit calulations are done using absolute time tracking. This means that the particle on the first turn enters into the RF cavity with a phase equal to zero, on subsequent turns the phase will increase. 
BMAD also has the ability to calculate dynamic aperture of a given lattice. The dynamic aperture is the maximum phase-space amplitude within which particles do not get lost as a consequence of single particle dynamics effect[1]. For proton rings, the concern is only the at injection due to the bunch adiabatically damping during acceleration. For simulations, the number of turns is 2000 .

\section{BMAD Comparison}

The lattices that were used for the simulations were parsed from lattices that were created in the MADX format using the Universal Accelerator Parser(UAP). The UAP has the ability to translate between Accelerator MarkUp Language (AML), BMAD, MAD8, and Extended Standard Input Format (XSIF). Radiofrequency cavities were added to the lattice to give a more realistic approach to the simulations. The species of the particle used in the two lattices that were parsed are proton and gold stripped of all electrons. The two lattices are injection with gamma equal to 25.379, and at store with gamma equal to 271.635 of the blue ring only for protons in Run 17. For gold, injection gamma is 10.520 and store gamma 107.3961 . The particle mass is taken to be $0.938 \mathrm{GeV}$ for protons and for gold, $183.43 \mathrm{GeV}$. The lattice length is $3833.845 \mathrm{~m}$. The number of magnet elements are exactly the same as the MADX lattice with the same s-coordinates.

The single particle and first turn tracking were implemented for both lattices. In using the parser, the strengths of all the quadrupole and dipole magnets within the MADX code are kept the same with the exception of the sextupole magnet strengths which were changed to provide for the lattice a chromaticity that is approximately 2 at both injection and store. The calculated natural chromaticity of the blue proton ring is -80.581 horizontal, and -15.953 vertical at injection and at store -70.697 and -63.413.For gold, the natural chromaticity is -84.563 horizontal, and -8.273 vertical at injection and at store -101.662 horizontal and -80.435 vertical. The natural chromaticity was found by setting all of the sextupole magnet strengths to zero.

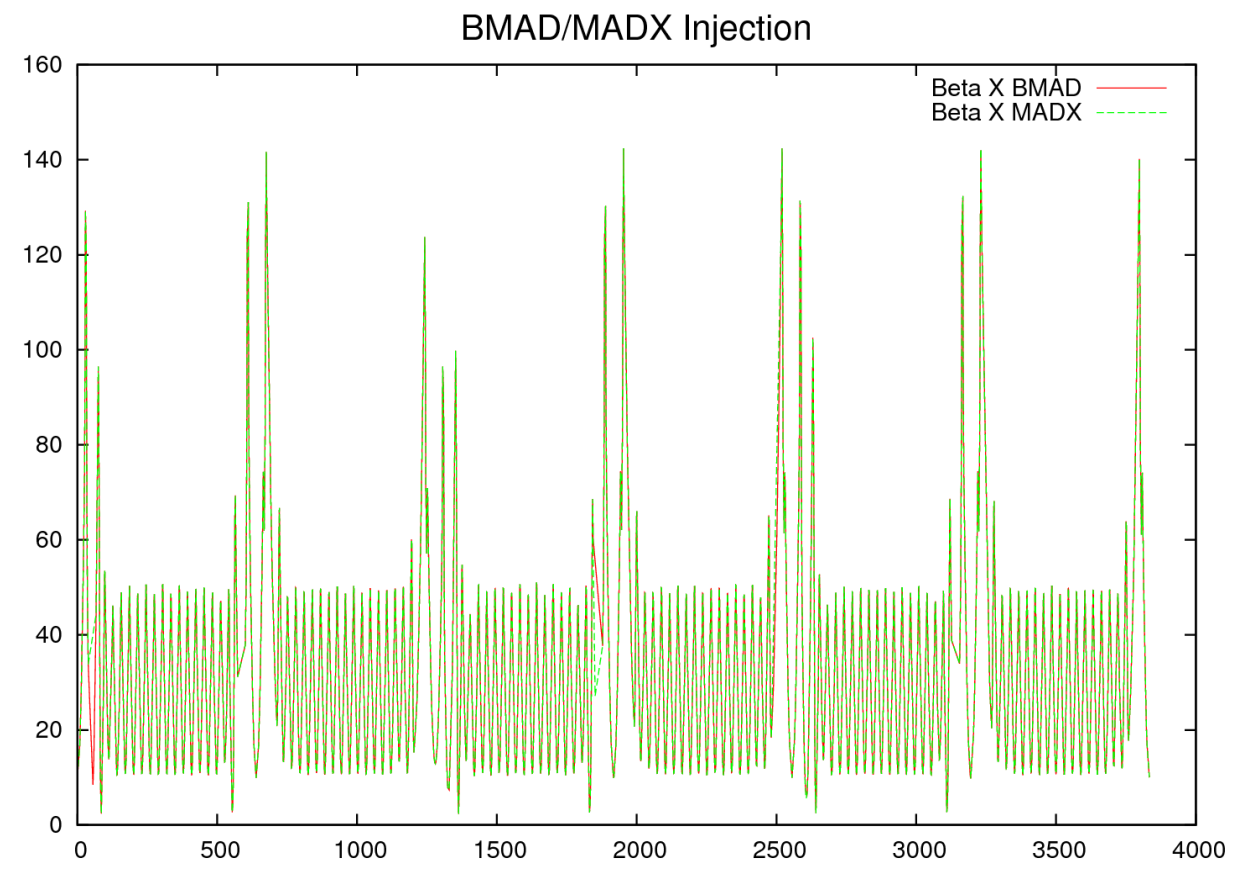

The betatron tunes at injection are 28.697, horizontal and 29.687, vertical. The amplitude of the beta function is taken at each element. 


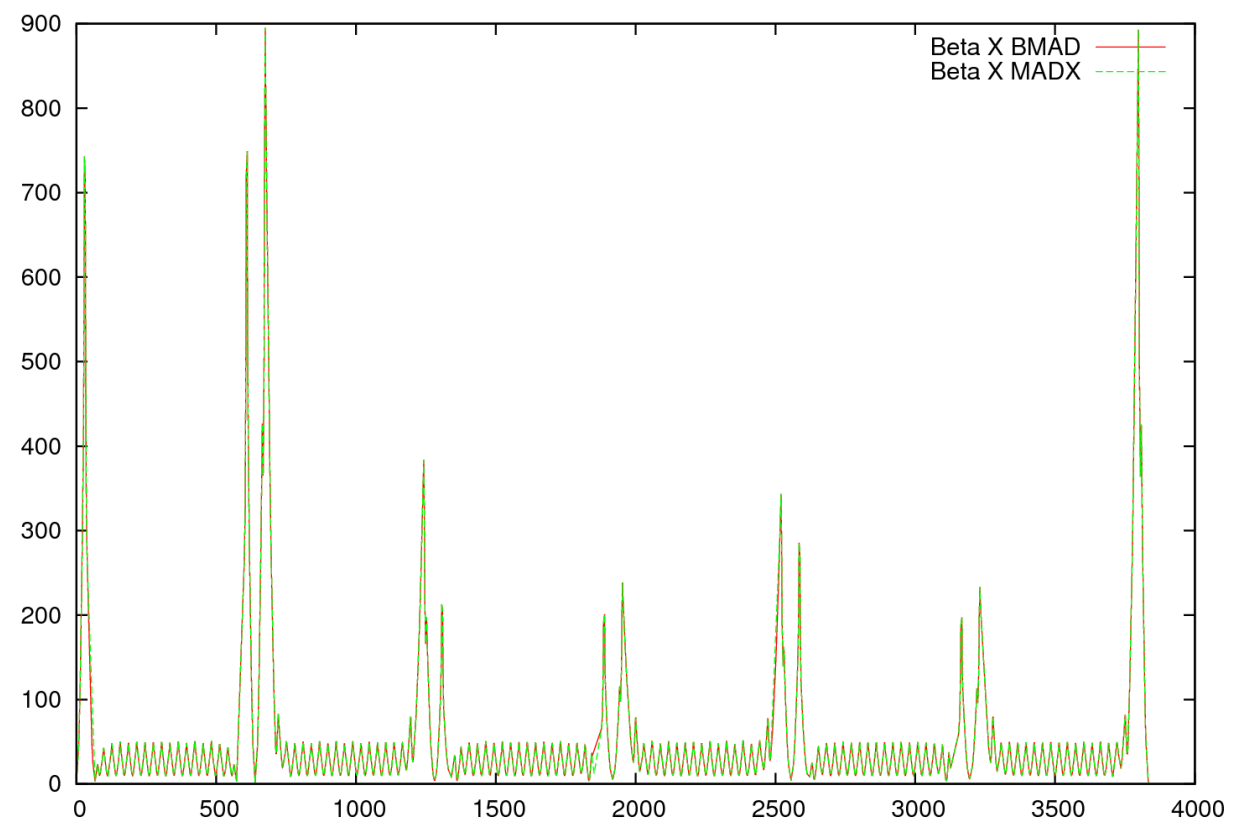

The tunes calculated at store are 28.689, horizontal and 29.684, vertical. The value for the tunes in both planes and for both lattices are in close agreement with the lattices that were used for parsing without retuning. With both tune calculations, at injection and at store, the error is less than $1 \%$. The percent error in the measurement of the horizontal beta amplitudes at the two IR 6 o'clock and 8 o'clock at store energy are both less than $1 \%, 1.62 * 10^{-3}$ and $2.36 * 10^{-3}$, respectively.

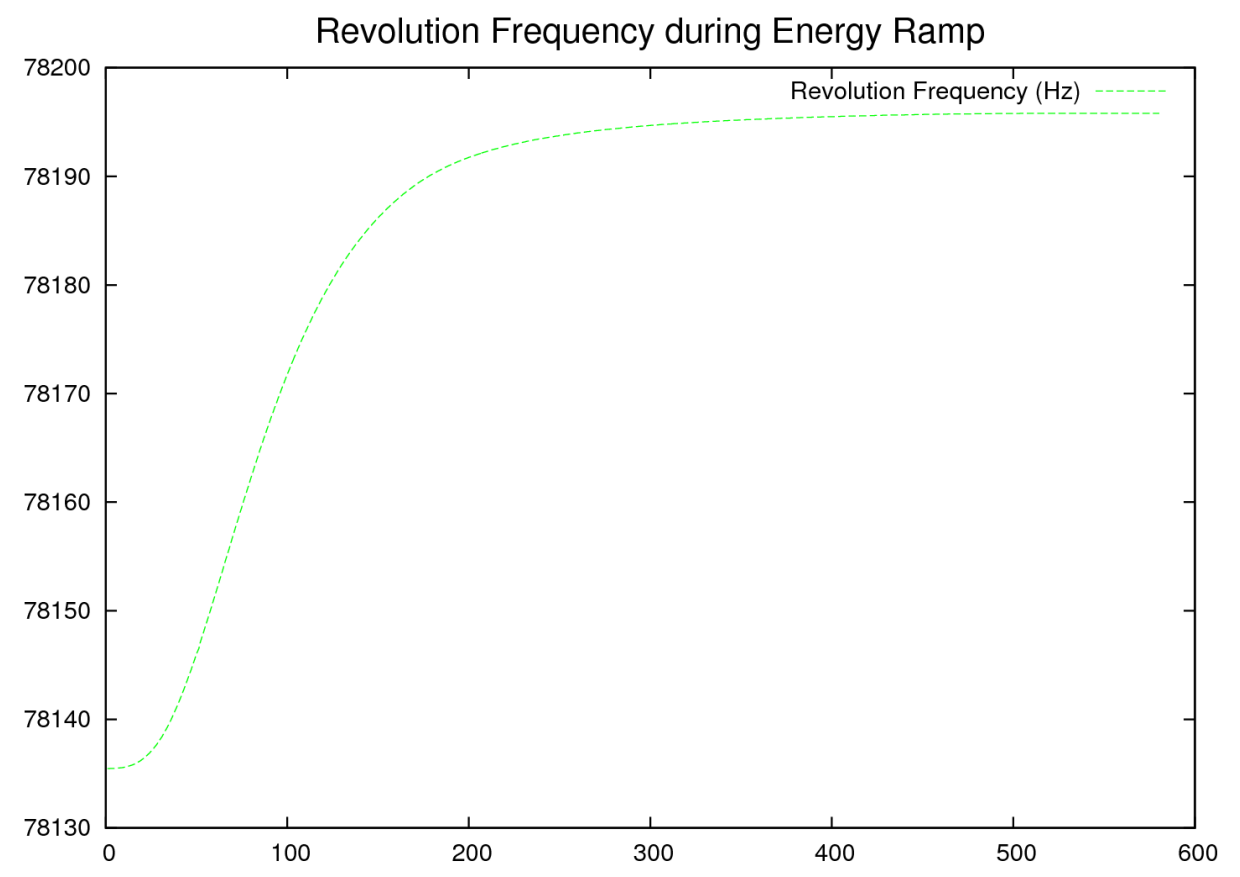

The BMAD calculated revolution frequency at injection is $78135.559 \mathrm{~Hz}$ and at store 78195.757 $\mathrm{Hz}$. The actual injection and store revolution frequency measurements are $78135.474 \mathrm{~Hz}$ and $78195.756 \mathrm{~Hz}$. The reason for the discrepencies between revolution frequency at injection is that the circumference is lengthened due to the helical dipoles for proton by $4.16 \mathrm{~mm}$ at injection. 


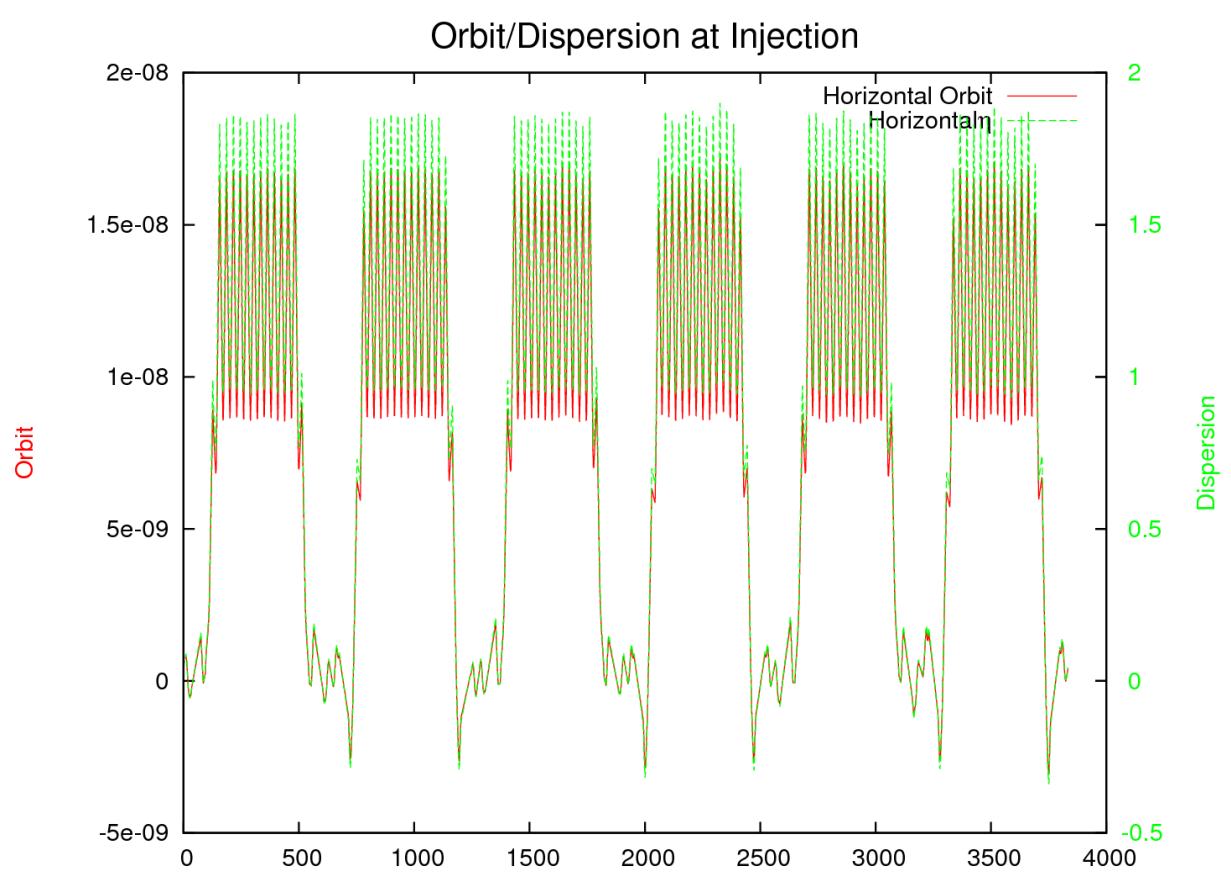

The lattices were improved with the inclusion of two RF gaps at injection and three at storage energy. These gaps represent the storage, acceleration, and Landau cavities used in ramping process. The three frequencies used in this process are $9.376 \mathrm{MHz}$ and $28.129 \mathrm{MHz}$ at injection, and 197.053 $\mathrm{MHz}$ is used for the storage gap. The corresponding harmonics are 120, 360, and 2520, respectively.

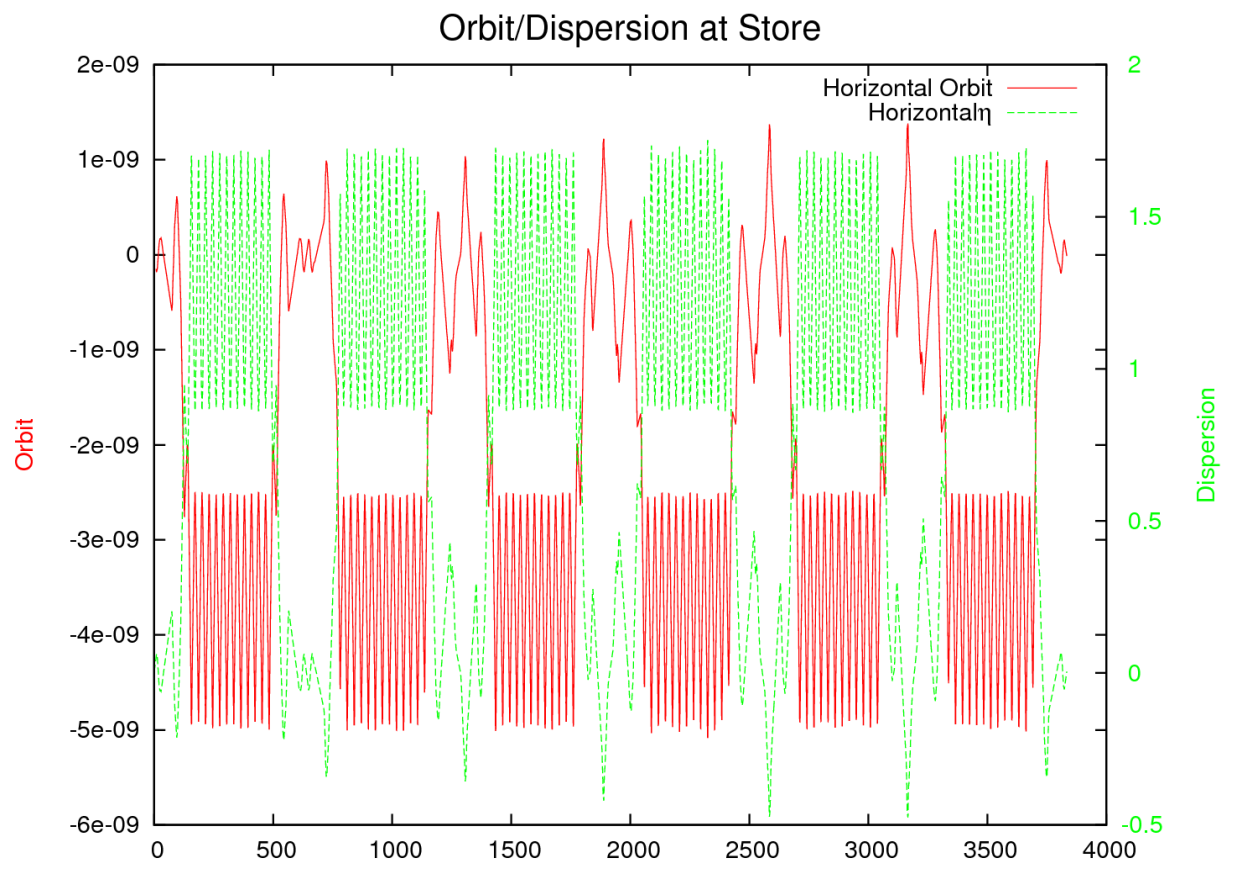

The effect of the RF on the close orbit is that in regions of zero dispersion the orbit excursion is at a minimum. The negative orbit excursion at store energy is due to negative momentum deviation. With increasing the energy at store or decreasing dipole magnet strength the orbit excursion can be made positive. 
For the gold lattice, the tunes at injection are 28.2441 horizontally and 29.2249 vertically are different from the MADX lattice tunes 28.2990 horizontally and 29.1634 vertically. This discrepency is due to diffences in the quadrupole strengths. With adjustments to the quadrupole strength the tunes can be made to match.

BMAD/MADX at Injection Gold

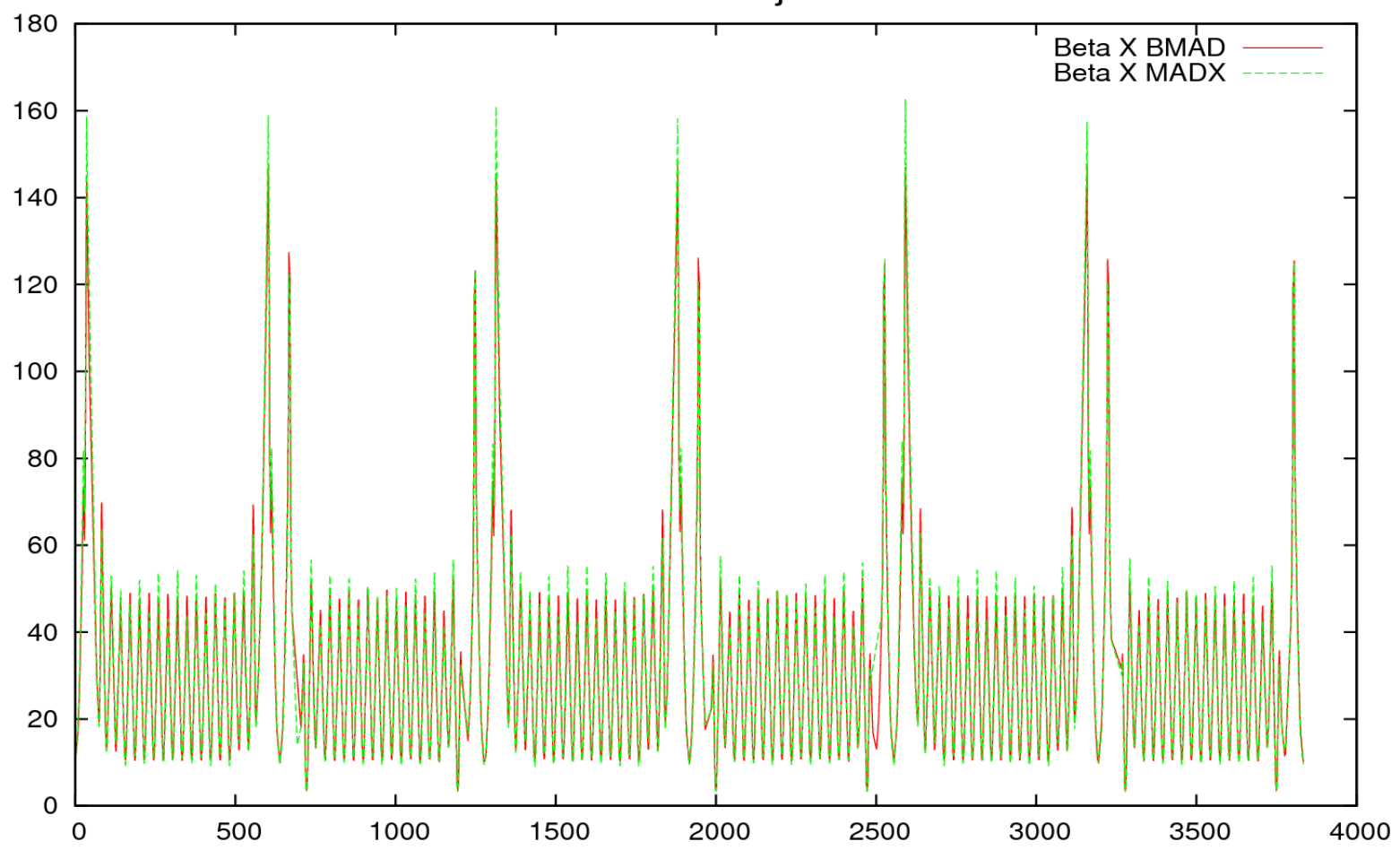

At store the tunes are 28.2420 horizontally and 29.21889 vertically. The MADX tunes are 28.2340 horizontally and 29.2280 vertically. There is a $-3.419 \%$ error between the fraction part of the horizontal tune and a 3.995\% error in fraction part of the vertical. As shown in both plots the beta amplitude calculated for gold at both injection and store closely match the results generated from MADX. The percent error in the measurement of the horizontal beta amplitudes at the two IR 6 o'clock and 8 o'clock at store energy are both less than 2\%, 1.777\% and 1.632\%, respectively. The increase in percent error, in comparison to the protons, is due to the tune difference. The transition gamma for the RHIC design lattice is 22.89 , for the calculated transition is 22.911 at injection energy which has an error less than a .1\%. 
BMAD/MADX at store Gold

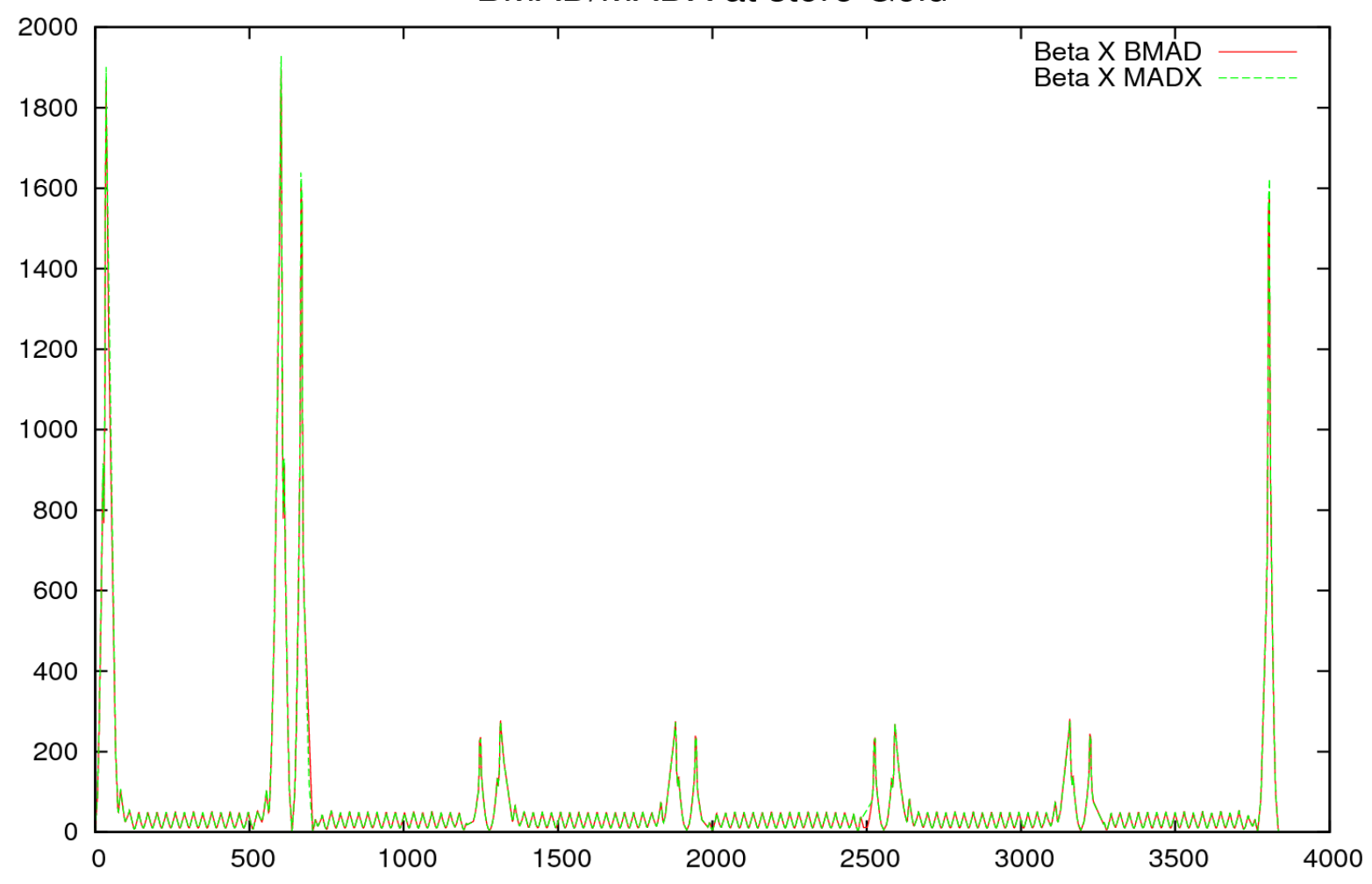

The BMAD calculated revolution frequency at injection is $77842.234 \mathrm{~Hz}$ and at store 78192.897 $\mathrm{Hz}$. The actual injection and store revolution frequency measurements are $77842.232 \mathrm{~Hz}$ and $78192.897 \mathrm{~Hz}$.

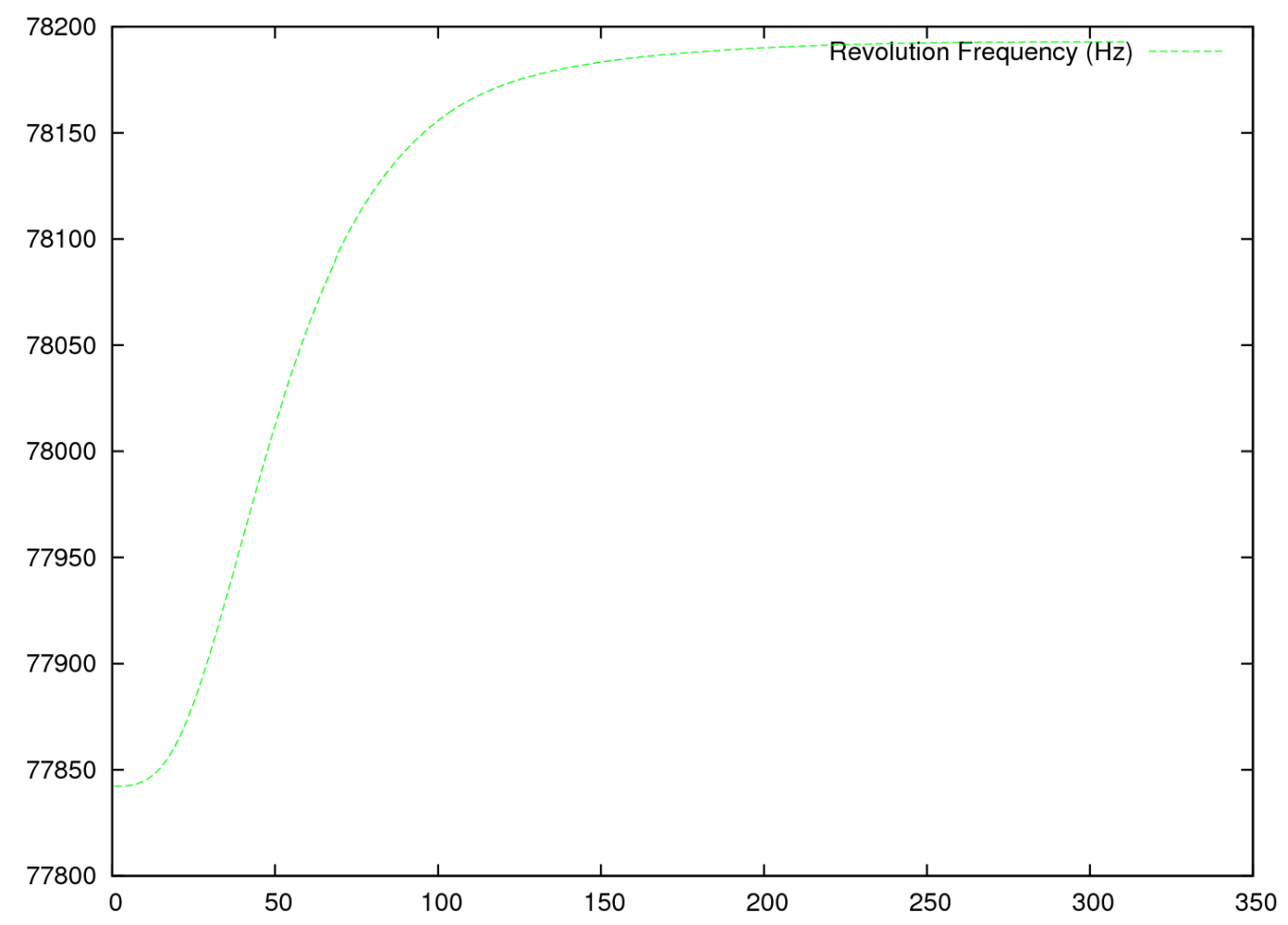

The gold lattices were improved with the inclusion of one RF gaps at injection and two at storage energy. These gaps represent the storage and acceleration cavities used in ramping process. The two frequencies used in this process are 28.129 MHz at injection, and 197.053 MHz at store. The 
corresponding harmonics are 360, and 2520, respectively.
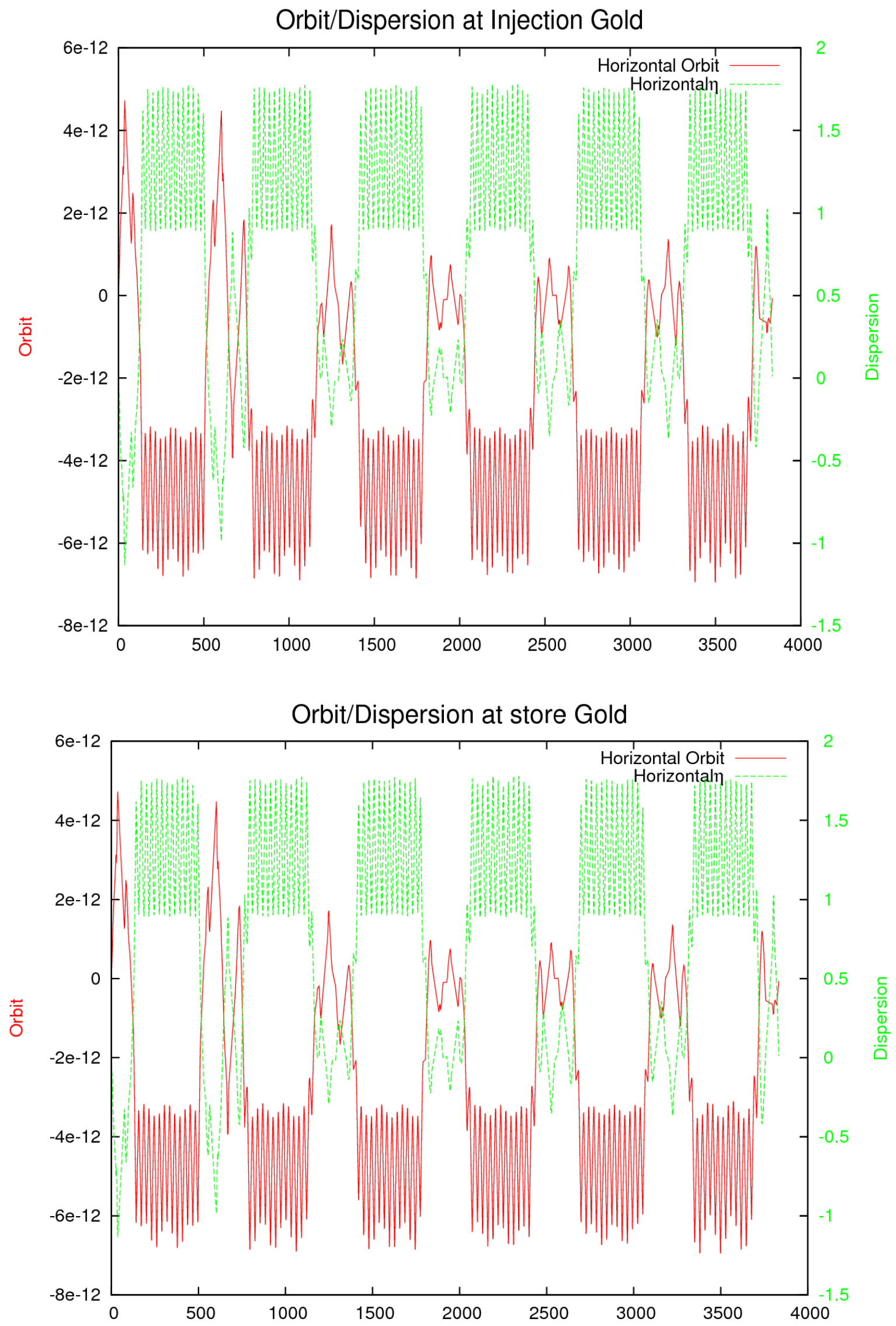

The phase space can also be studied using BMAD code. Multi-turn tracking calulation of the 6 dimensional phase space can be made where an initial vector can be given and a number of turns 
specified. Phase ellipse focusing and defocusing can be demonstrated. In the first plot, a single particle is plotted for 1000 turns through the focusing quadrupole YO1_QF20, for the second plot the particle is plotted through the defocusing quadrupole YO1_QD11. Both plots are at injection energy.
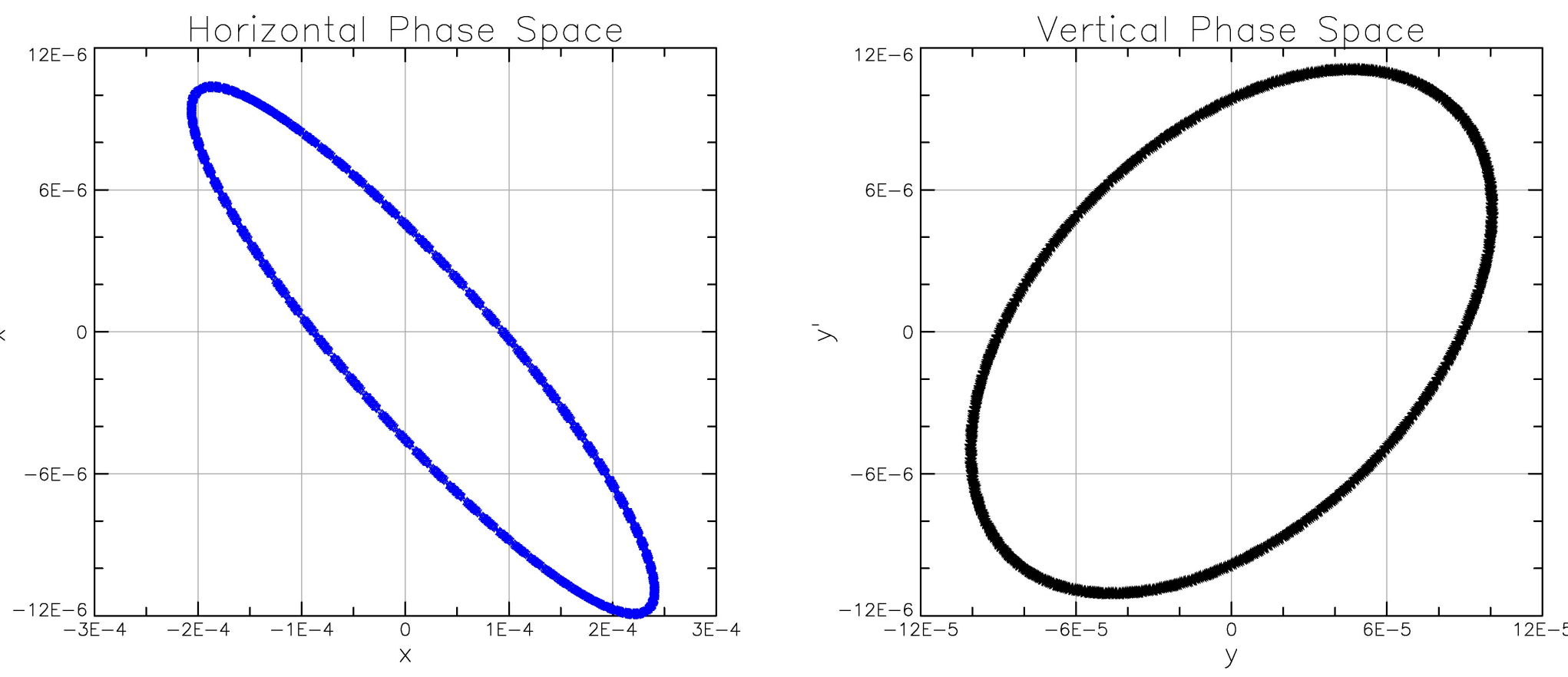

phase_space.dat
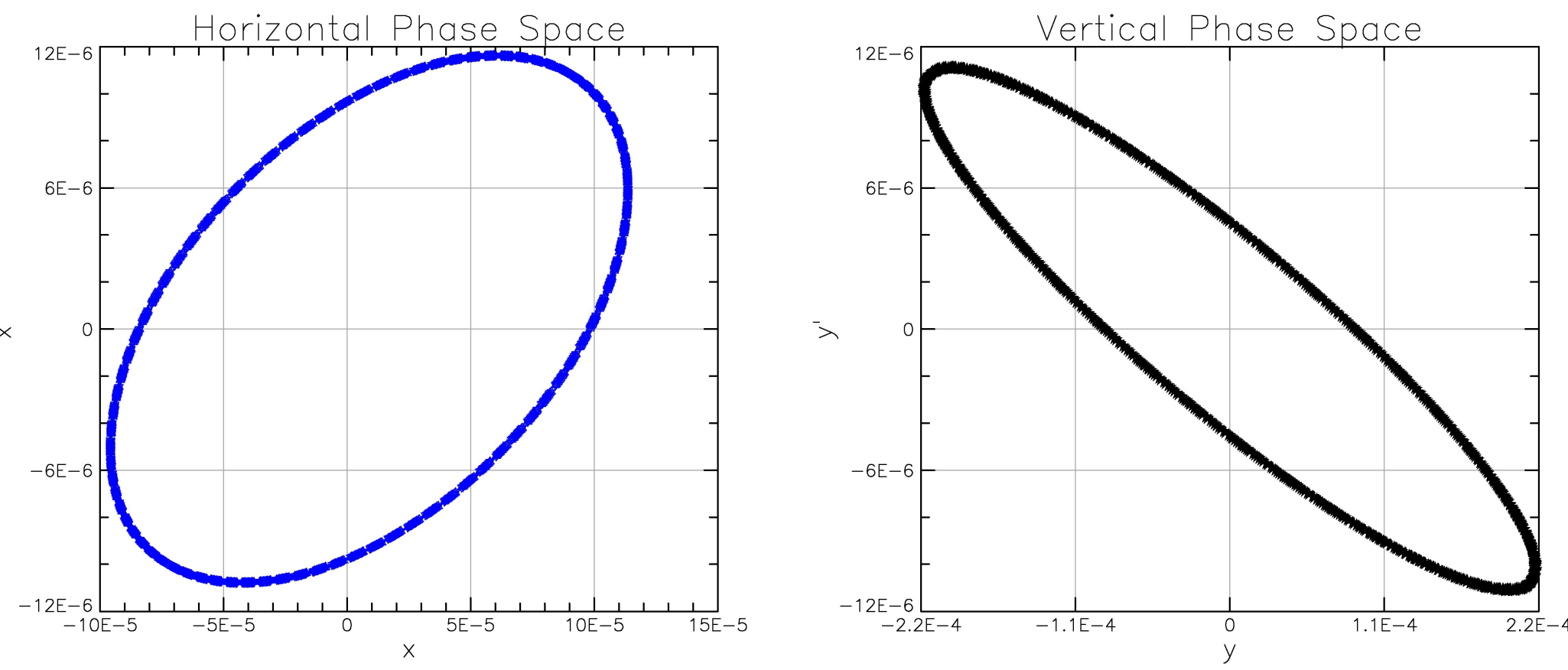

In RHIC, the first 1024 turns are tracked to give an approximation of the number of amplitude of the oscillations taken in a turn by turn method. A maximum amplitude plot can be created with a given initial amplitude. An energy difference is given for each plot. The initial amplitude given to the proton is $.5 \mathrm{~mm}$ in both planes. The points are generated when the particle becomes lost within the 2000 turns that are tracked with the RF off. 


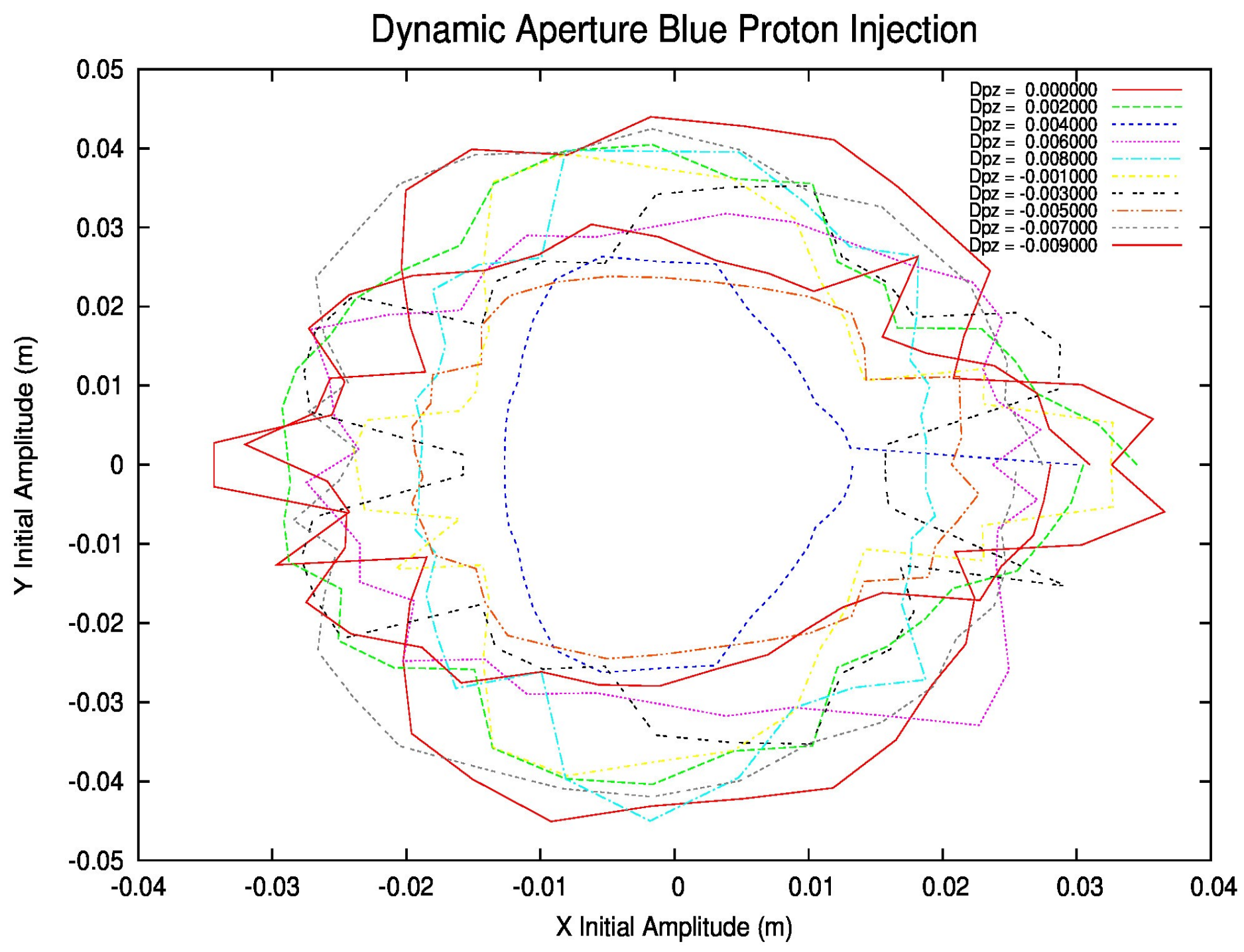

Conclusion

A comparison between BMAD and MADX simulations is useful. The power of BMAD in creating, modeling, and particle tracking through a lattice configuration was demonstrated. The close approximation in the results for lattice modeling show that BMAD may be implented as a stand alone program in future lattice designs. The percent error in the measurement of the horizontal beta amplitudes at the two IR, 6 o'clock and 8 o'clock, at store energy for protons are $1.62 * 10^{-3} \%$ and $2.36 * 10^{-3} \%$, respectively. For gold, the percent error of the horizontal beta amplitudes at the two IR are less than $2 \%, 1.777 \%$ and $1.632 \%$. The increased percent error for the beta amplitudes IR is due to the $-3.419 \%$ error horizontally. If the tunes were matched completely as in the proton lattice, then the percent error would be less than $1 \%$. The single particle tracking through specific elements in a lattice allows for the study of the development of the beam envelope over a given amount of turns. Amplitude plots can be created using BMAD for protons with particle survival being the point generator. 


\section{Acknowledgements}

I would like to thank Michael Blaskiewicz for the patience that it takes to be a consultant on this benchmarking adventure. Steve Tepikian for his helpful insight on the RHIC lattice. Christopher Gardener for giving advice on providing the parameters of the RHIC lattices and David Sagan for creating the BMAD modules and advice given on this paper.

Bibliograpy

“Bmad Home Page.” Bmad Home Page, www.lepp.cornell.edu/ dcs/bmad. Accessed 14 Apr. 2017.

Chao, Alexander Wu. Handbook of Accelerator Physics and Engineering. Singapore, World Scientific Publishing, 1999.

Forest , Etienne. Implementation and Illustration of Perturbation Theory in a Tracking Code . Springer , 2014

“The Tao Manual.” www.lepp.cornell.edu/ dcs/bmad/tao entry point.html. Accessed 14 Apr. 2017. 
BMAD

\begin{tabular}{|c|c|c|c|c|}
\hline Lattice Parameter & Protons injection & Protons store & Gold injection & Gold store \\
\hline$\gamma$ & 25.37862 & 271.6349 & 10.52046 & 107.3960 \\
\hline$v_{\mathrm{h}}$ & 28.69700 & 28.68878 & 28.24416 & 28.24203 \\
\hline$v_{\mathrm{v}}$ & 29.68700 & 29.684020 & 29.22492 & 29.21889 \\
\hline$\xi_{\mathrm{h}}$ & 2.08977 & 2.01335 & 2.0146 & 2.0057 \\
\hline$\xi_{\mathrm{v}}$ & 2.084395 & 2.00643 & 2.0259 & 2.0074 \\
\hline$\xi_{\mathrm{h}}{ }^{\text {nat }}$ & -80.581 & -70.697 & -84.563 & -101.662 \\
\hline$\xi_{\mathrm{v}}{ }^{\text {nat }}$ & -15.953 & -63.413 & -8.273 & -80.435 \\
\hline$\beta_{\mathrm{h}}{ }^{\max }$ & 142.4171 & 894.9579 & 147.0132 & 1895.4639 \\
\hline$\beta_{\mathrm{v}}{ }^{\max }$ & 142.1800 & 916.5739 & 150.5372 & 1874.1010 \\
\hline$\eta_{\mathrm{h}}{ }^{\max }$ & 1.8991 & 1.7505 & 1.8194 & 1.8237 \\
\hline$\eta_{\mathrm{v}}{ }^{\max }$ & 0 & 0 & 0 & 0 \\
\hline Rev. frequency & $78135.5588 *$ & 78195.7569 & 77842.2325 & 78192.8973 \\
\hline
\end{tabular}

*circumference lengthening not accounted for in calculation

MADX

\begin{tabular}{|c|c|c|c|c|}
\hline Lattice Parameter & Protons injection & Protons store & Gold injection & Gold store \\
\hline$\gamma$ & 25.37862 & 271.6349 & 10.52046 & 107.3960 \\
\hline$v_{\mathrm{h}}$ & 28.69700108 & 28.68878855 & 28.29902955 & 28.2339528 \\
\hline$v_{\mathrm{v}}$ & 29.68700054 & 29.68402021 & 29.16344748 & 29.22804679 \\
\hline$\xi_{\mathrm{h}}$ & 2.246452883 & 2.248794477 & -7.885572288 & 2.285092098 \\
\hline$\xi_{\mathrm{v}}$ & 2.083578948 & 2.09939207 & -6.015872636 & 2.11034623 \\
\hline$\beta_{\mathrm{h}}{ }^{\max }$ & 142.4170621 & 894.9569606 & 162.6747942 & 1928.242576 \\
\hline$\beta_{\mathrm{v}}{ }^{\max }$ & 142.1800042 & 916.5740045 & 153.4966541 & 1900.295911 \\
\hline$\eta_{\mathrm{h}}{ }^{\max }$ & 1.900555941 & 1.750497005 & 1.829091458 & 1.795521833 \\
\hline$\eta_{\mathrm{v}}{ }^{\max }$ & 0 & 0 & 0 & 0 \\
\hline Rev. frequency* & 78135.474 & 78195.757 & 77842.232 & 78192.897 \\
\hline
\end{tabular}

\footnotetext{
* Logview Application
} 
Appendix

Derivation Courant-Snyder invariant using phasor notation

$$
\begin{aligned}
& F\left(z_{1}, z_{2}\right) \quad \text { one degree of freedom } \\
& \langle F\rangle=\frac{\left(F \circ m+F \circ m \circ m+\ldots+F \circ m^{N}\right)}{N} \text { where } N \rightarrow \infty \\
& m \equiv \text { one turn map } \\
& m=a \circ r \circ a^{-1} \\
& r\left(\begin{array}{l}
z_{1} \\
z_{2}
\end{array}\right)=\left[\begin{array}{ll}
\cos \mu(J) & \sin \mu(J) \\
\sin \mu(J) & \cos \mu(J)
\end{array}\right]\left(\begin{array}{l}
z_{1} \\
z_{2}
\end{array}\right) \text { where } 2 J=z_{1}^{2}+z_{2}^{2} \\
& \langle F\rangle=\frac{\left(F \circ a \circ r^{0} \circ a^{-1}+F \circ a \circ r \circ a^{-1}+F \circ a \circ r^{2} \circ a^{-1}+\ldots+F \circ\left\{a \circ r \circ a^{-1}\right\}\right)^{N}}{N} \text { where } N \rightarrow \infty \\
& \langle F\rangle=\frac{\left(F \circ \boldsymbol{a} \circ r^{0}+F \circ \boldsymbol{a} \circ \boldsymbol{r}+F \circ \boldsymbol{a} \circ \boldsymbol{r}^{2}+\ldots+F \circ \boldsymbol{a} \circ r^{N}\right)}{N} \circ a^{-1} \text { where } N \rightarrow \infty \\
& \langle\bar{F}\rangle=\frac{\left(\bar{F} \circ a \circ r^{0}+\bar{F} \circ a \circ r+\bar{F} \circ a \circ r^{2}+\ldots+\bar{F} \circ a \circ r^{N}\right)}{N} \circ a^{-1} \text { where } N \rightarrow \infty \\
& \bar{F}=F \circ a \\
& \bar{F} \circ r^{k} \circ c=\{\bar{F} \circ c\} \circ\left\{c^{-1} \circ r^{k} \circ c\right\}=F^{r} \circ \Lambda \\
& \Lambda \equiv \text { diagonalized rotation }
\end{aligned}
$$

The resonance basis is

$$
\begin{gathered}
\left(\begin{array}{l}
r_{1} \\
r_{2}
\end{array}\right)=c\left(\begin{array}{l}
z_{1} \\
z_{2}
\end{array}\right)=\left(\begin{array}{cc}
\frac{1}{2} & \frac{1}{2} \\
\frac{-i}{2} & \frac{-i}{2}
\end{array}\right)\left(\begin{array}{l}
z_{1} \\
z_{2}
\end{array}\right) \\
c^{-1}\left(\begin{array}{l}
z_{1} \\
z_{2}
\end{array}\right)=\left(\begin{array}{cc}
1 & i \\
1 & -i
\end{array}\right)\left(\begin{array}{l}
z_{1} \\
z_{2}
\end{array}\right) \\
F^{r}=\sum F_{m n}^{r} r_{1}^{m} r_{2}^{n} \\
F^{r} \circ \Lambda
\end{gathered}
$$

Example:

$$
\begin{gathered}
\left(\begin{array}{l}
z_{1} \\
z_{2}
\end{array}\right)=\left(\begin{array}{cc}
\sqrt{\beta} & 0 \\
\frac{-\alpha}{\sqrt{\beta}} & \frac{1}{\sqrt{\beta}}
\end{array}\right)\left(\begin{array}{l}
x \\
p
\end{array}\right)=a\left(\begin{array}{l}
x \\
p
\end{array}\right) \\
a^{-1}\left(\begin{array}{l}
x \\
p
\end{array}\right)=\left(\begin{array}{cc}
\frac{1}{\sqrt{\beta}} & 0 \\
\frac{\alpha}{\sqrt{\beta}} & \sqrt{\beta}
\end{array}\right)\left(\begin{array}{l}
x \\
p
\end{array}\right) \\
\bar{F} \circ r^{k} \circ c=\{\bar{F} \circ c\} \circ\left\{c^{-1} \circ r^{k} \circ c\right\}=F^{r} \circ \Lambda \\
F^{r}=\bar{F} \circ c \sqrt{\beta} \circ c=\beta \frac{\left(r_{1}^{2}+r_{2}^{2}+2 r_{1} r_{2}\right)}{4}
\end{gathered}
$$

For the average,

$$
\begin{gathered}
\left\langle F^{r}\right\rangle=\beta \frac{\left(2 r_{1} r_{2}\right)}{4}=\frac{\beta}{2}\left(r_{1} r_{2}\right) \\
\langle\bar{F}\rangle=\frac{\beta}{2}\left(r_{1} r_{2}\right) \circ c^{-1}=\frac{\beta}{2}\left(z_{1}^{2}+z_{2}^{2}\right)=\beta J \\
\text { where } \quad r_{1}=z_{1}+z_{2} i \text { and } r_{1}=z_{1}+z_{2} i \\
\langle F\rangle=\beta J \circ a^{-1}=\frac{\beta}{2}\left(z_{1}^{2}+z_{2}^{2}\right) \circ a^{-1}
\end{gathered}
$$




$$
\begin{gathered}
\langle F\rangle=\frac{\beta}{2}\left(\frac{1}{\beta}+\frac{\alpha^{2}}{\beta} x^{2}+2 \alpha x p+\beta p^{2}\right) \\
\langle F\rangle=\frac{\beta}{2}\left(\gamma x^{2}+2 \alpha x p+\beta p^{2}\right)
\end{gathered}
$$

Let,

$$
\begin{aligned}
& M=\left(\begin{array}{ll}
a & b \\
c & d
\end{array}\right) \text { where } a d-b c=1 \\
& M=S R S^{-1} \\
& R=\left(\begin{array}{cc}
\cos \mu & \sin \mu \\
-\sin \mu & \cos \mu
\end{array}\right) \quad S=\left(\begin{array}{cc}
S_{1} & S_{12} \\
S_{21} & S_{22}
\end{array}\right) \quad S^{-1}=\left(\begin{array}{cc}
S_{22} & -S_{12} \\
-S_{21} & S_{11}
\end{array}\right) \\
& \operatorname{det} S=S_{11} S_{22}-S_{12} S_{21}=1 \\
& M=\left(\begin{array}{ll}
S_{11} & S_{12} \\
S_{21} & S_{22}
\end{array}\right)\left(\begin{array}{cc}
\cos \mu & \sin \mu \\
-\sin \mu & \cos \mu
\end{array}\right)\left(\begin{array}{cc}
S_{22} & -S_{12} \\
-S_{21} & S_{11}
\end{array}\right) \\
& M=\left(\begin{array}{cc}
S_{11} S_{22} \cos \mu-S_{11} S_{21} \sin \mu-S_{12} S_{22} \sin \mu-S_{12} S_{21} \cos \mu & -S_{11} S_{12} \cos \mu+S_{11}^{2} \sin \mu+S_{12}^{2} \sin \mu+S_{12} S_{11} \cos \mu \\
S_{21} S_{22} \cos \mu-S_{21}^{2} \sin \mu-S_{22}^{2} \sin \mu-S_{21} S_{22} \cos \mu & -S_{12} S_{21} \cos \mu+S_{21} S_{11} \sin \mu+S_{22} S_{12} \sin \mu+S_{11} S_{22} \cos \mu
\end{array}\right) \\
& M=\left(S_{11} S_{22}-S_{12} S_{21}\right) \cos \mu \bar{I}+\sin \mu\left(\begin{array}{cc}
-S_{11} S_{21}-S_{12} S_{22} & S_{11}^{2}+S_{12}^{2} \\
-S_{21}^{2}-S_{22}^{2} & S_{21} S_{11}+S_{22} S_{12}
\end{array}\right) \\
& M=\cos \mu \bar{I}+\sin \mu\left(\begin{array}{cc}
-S_{11} S_{21}-S_{12} S_{22} & S_{11}^{2}+S_{12}^{2} \\
-S_{21}^{2}-S_{22}^{2} & S_{21} S_{11}+S_{22} S_{12}
\end{array}\right) \\
& M=\cos \mu \bar{I}+\sin \mu\left(\begin{array}{cc}
\alpha & \beta \\
-\gamma & -\alpha
\end{array}\right) \\
& r^{2}=z_{1}^{2}+z_{2}^{2} \\
& \epsilon(x, p)=r^{2}(z(x, p))=\left(s_{1}^{-1} z(x, p)\right)^{2}+\left(s_{2}^{-1} z(x, p)\right)^{2} \\
& \epsilon=\left(S_{22} x-S_{12} p\right)^{2}+\left(-S_{21} x+S_{11} p\right)^{2} \\
& \epsilon=\left(S_{22}^{2}+S_{21}^{2}\right) x^{2}+\left(S_{12}+S_{11}^{2}\right) p^{2}+2\left(-S_{22}^{2} S_{12}-S_{21} S_{11}\right) x p
\end{aligned}
$$


RHIC LATTICE

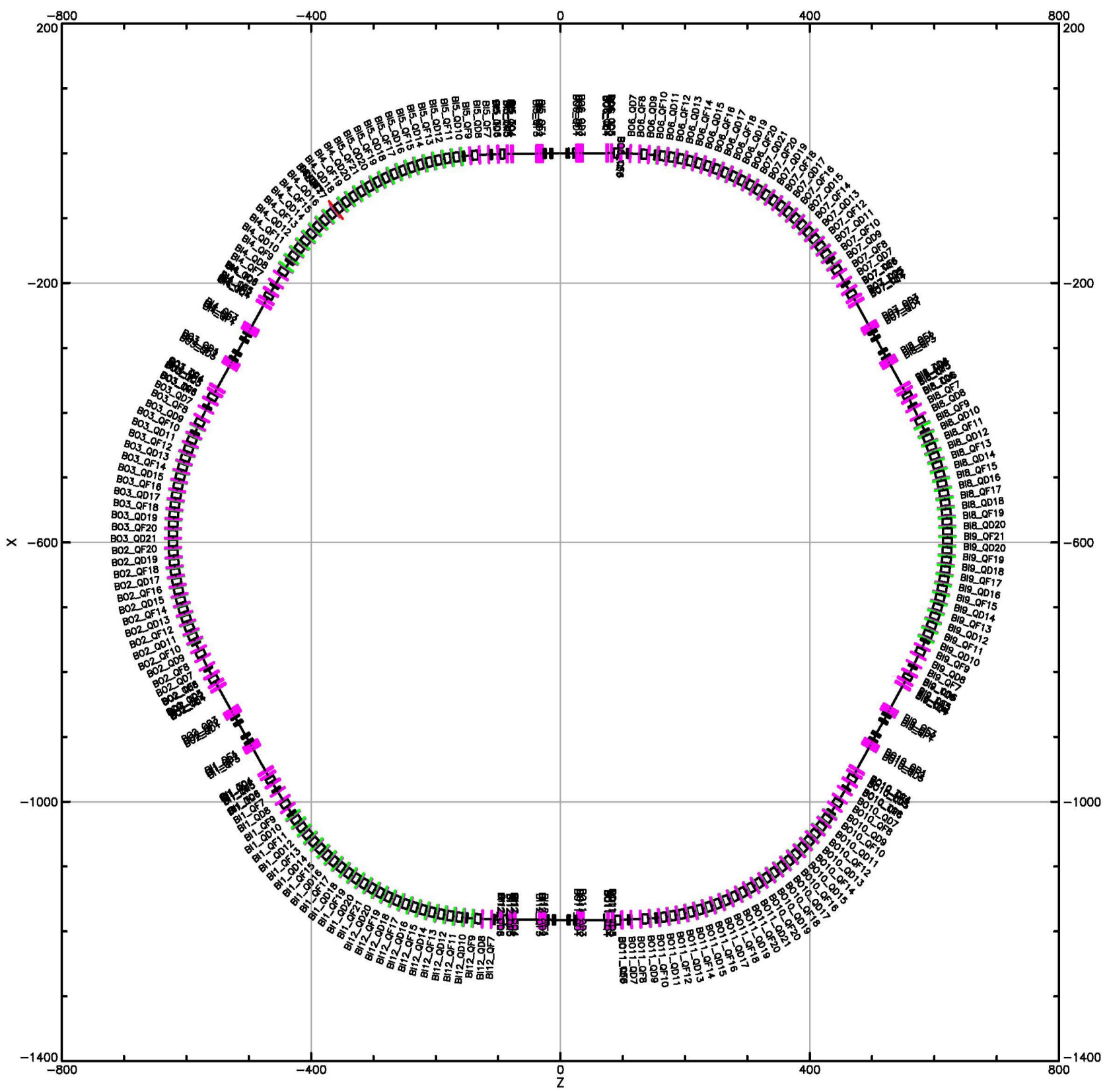


Typical Print out of BMAD program

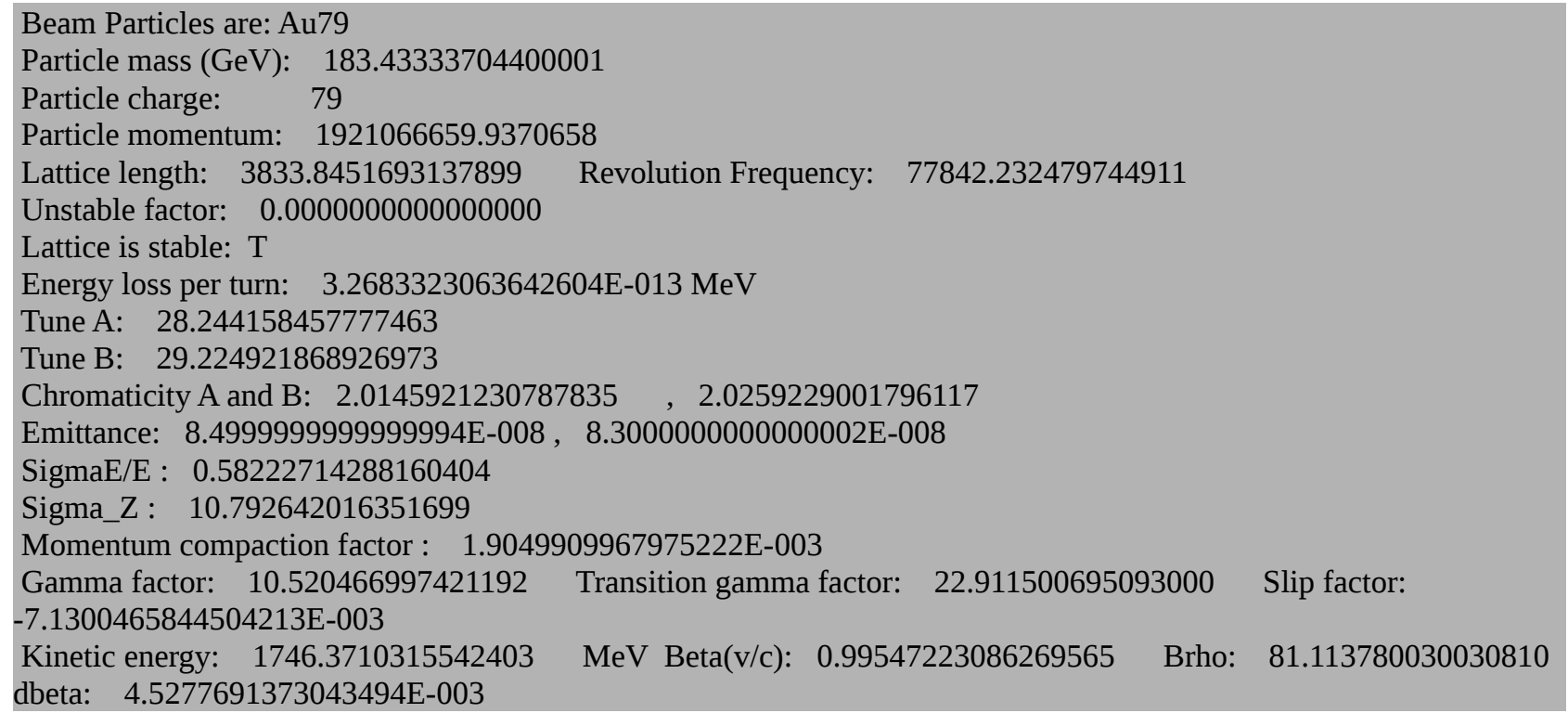

!Note!

Emittances, SigmaE/E, and Sigma_Z are given as parameters to the program not calculated. 